Pacific Journal of Mathematics

A CONTINUITY PROPERTY FOR VECTOR VALUED 


\title{
A CONTINUITY PROPERTY FOR VECTOR VALUED MEASURABLE FUNCTIONS
}

\author{
R. B. DARST
}

1. Introduction. The principal purpose of this paper is to characterize certain types of Banach spaces of vector valued and integrable functions and their conjugate or adjoint spaces, and to apply these characterizations to obtain an effective way of determining the adjoint space of the Banach space $R[a, b]$, where $[a, b]$ is a real number interval, $R[a, b]$ is the space of Riemann integrable functions on $[a, b]$, and for $f \in R[a, b],\|f\|=\sup [|f(x)| ; a \leqq x \leqq b]$. We use the term effective in the sense that we want our determination of the adjoint space of $R[a, b]$ to enable us to probe deeper into its analytic structure in order to, say for example, obtain a weak convergence and compactness theory.

We are interested in spaces of vector valued functions which arise in the following manner. Let $X$ be a set, $B$ a Banach space, and $f$ a a vector valued function on $X$ to $B$. We call $f$ partitionable if for each $\varepsilon>0$, there exists a finite partition $\left[E_{i} ; i \leqq n\right]$ of $X$ such that $\max \left[0\left(f, E_{i}\right) ; i \leqq n\right]<\varepsilon$, where $0\left(f, E_{i}\right)=\sup \left[\|f(x)-f(y)\| ; x, y \in E_{i}\right]$. Hence, if $S$ is an algebra of subsets of $X$ and $g$ is a bounded and finitely additive set function on $S$, then the collection of bounded, $B$ valued, partitionable, and $g$-measurable functions on $X$ (defined in $\S 2$ ) form a Banach space which we denote by $m(X, S, g, B)$ (the norm of the elements $f$ of $m(X, S, g, B)$ is $\sup [\|f(x)\| ; x \in X])$. The space $m(X, S, g, B)$ is the type of space we characterize and we do it by isomorphically and isometrically embedding $m(X, S, g, B)$ onto the space $C(X, T, B)$ of $B$-valued functions defined on $X$ which are continuous (Definition 2.4) with respect to an algebra $T$ of subsets of $X$. The set $T$ is essentially the $g$-outer measure completion of $S$. Then we characterize the adjoint space $C^{*}(X, T, B)$ of the space $C(X, T, B)$ by embedding $C^{*}(X, T, B)$ isomorphically and isometrically onto a space of bounded $B^{*}$-valued finitely additive set functions on $T$ (defined in $\S 3$ ) and, consequently, we can easily find the adjoint space $m^{*}(X, S, g, B)$. In the case where $B$ is the real number system (henceforth we shall denote by $\mathscr{R}$ the real number system) we simply denote $m(X, S, g, B)$ by $m(X, S, g)$. The space $R[a, b]$ turns out to be a realization of this type of space and it is by this method that we characterize $R^{*}[a, b]$.

The text of this paper consists of three sections. In $\S 2$ we present several definitions and introduce some notation and terminology. In $\S 3$ our principal results (Theorems 3.2, 3.3, and 3.4) give a characterization of the spaces $m(X, S, g, B), C^{*}(X, S, B)$, and $m^{*}(X, S, g, B)$ respectively,

Received June 2, 1961. 
and in $\S 4$ we treat the space $R[a, b]$.

At this point we wish to remind the reader that if $B$ is finite dimensional and $f$ is a bounded $B$-valued function on $X$ then $f$ is partitionable.

2. Definitions, notation, and terminology. We shall use the notation and terminology used in [1]. Hence, if $(X, S)$ denotes a set algebra, then $H(X, S)$ denotes the Banach space of bounded and finitely additive set functions on $S$, where the norm of the elements $g$ of $H(X, S)$ is the total variation $V(g, X)$ of $g$ on $X$, i.e., if for the elements $E$ of $S$, we set $g^{+}(E)=\sup [g(F) ; F \in S, F \subset E]$ and $g^{-}(E)=\inf [g(F) ; F \in S$, $F \subset E]$, then $V(g, E)=g^{+}(E)-g^{-}(E)=\sup \left[\sum_{1 \leqq n}\left|g\left(E_{i}\right)\right| ; \pi=\left[E_{i} ; i \leqq n\right]\right.$ is a partition of $E$ and $E_{i} \in S$ for $\left.i \leqq n\right]$.

Definition 2.1. If $U$ is a set of subsets of $X$, then a partition $\pi$ of $X$ is said to be a partition of $X$ in $U$ if each element of $\pi$ is an element of $U$.

In this paper we use a definition of measurability which is for bounded functions equivalent to the definition of measurability used in [2].

Definition 2.2. Suppose $(X, S)$ is a set algebra, $g \in H(X, S), B$ is a Banach space, and $f$ is a function on $X$ to $B$. Then $f$ is said to be a $g$-measurable function if for each $P>0$ and $\varepsilon>0$ there exists a finite partition $\left[E_{i} ; i \leqq n\right]$ of $X$ in $S$ such that

(1) $\max \left[0\left(f^{p}, E_{i}\right) ; i<n\right]<\varepsilon$ and

(2) $V\left(g, E_{n}\right)<\varepsilon$, where $f^{P}(x)=f(x)$ if $\|f(x)\| \leqq P$ and $f^{P}(x)=$ $P \cdot f(x) /\|f(x)\|$ if $\|f(x)\|>P$.

Definition 2.3. A function $f$ on $X$ to $B$ is said to be a $(X, S, B)$ simple function if (1) the range of $f$ is a finite subset of $B$ and (2) for each $b \in B, f^{-1}(b) \in S$.

Definition 2.4. A function $f$ on $X$ to $B$ is said to be a $(X, S, B)$ continuous function if for each $\varepsilon>0$, there exists a finite partition $\left[E_{i} ; i \leqq n\right]$ of $X$ in $S$ such that $\max \left[0\left(f, E_{i}\right) ; i \leqq n\right]<\varepsilon$ (cf. [1]). We shall denote by $C(X, S, B)$ the Banach space of $(X, S, B)$-continuous functions.

REMARK. We note (1) that $C(X, S, B)$ is isomorphically isometric, via the natural embedding, to the Banach space of topologically continuous $B$-valued functions defined on the Stone-Cech type compactification of $S$, (2) that a function $f$ on $X$ to $B$ is in $C(X, S, B)$ if and only if for each $\varepsilon>0$, there exists a $(X, S, B)$-simple function $k$ such 
that $\|k-f\|=\sup [\|k(x)-f(x)\| ; x \in X]<\varepsilon: C(X, S, B)$ is the completion of the normed linear space of $(X, S, B)$-simple functions, (3) that if a $B$-valued function $f$ on $X$ is not partitionable, then for no algebra $W$ of subsets of $X$ is it possible to uniformly approximate $f$ by $(X, W, B)$ simple functions: if there exists an algebra $W$ of subsets of $X$ such that $f \in C(X, W, B)$ then $f$ is partitionable, and (4) that $C(X, S, B)=$ $\bigcap_{g \in H(X, S)} m(X, S, g, B)$ (cf. [1], Theorem 2.1).

3. The principal theorems. In this section we establish our principal results (Theorems 3.2, 3.3, and 3.4). However, in order to relate our definition of measurability to classical results we shall first digress for a few moments.

LEMMa 3.1. Let $f$ be a bounded B-valued function on $X, g \in H(X, S)$, and $f$ be Stieltjes integrable with respect to $g$ (cf. [5]), i.e., $\int_{X} f d g$ exists, then $f$ is Stieltjes integrable with respect to $V(g$,$) .$

Proof. It is sufficient to show that $\int_{X} f d g^{+}$exists. (This may be seen as follows. Suppose $\int_{X} f d g^{+}$exists. Then, since $g^{-}=(-g)^{+}, \int_{X} f d g^{-}$ exists and, hence, since $V(g)=,g^{+}-g^{-}, \int_{X} f d V(g, \quad)$ exists.) Suppose $\varepsilon>0$. Then there exists $E \in S$ such that $g^{+}(X)-g(E)<\varepsilon / 2$ and, hence, $V\left(g^{\div}-g / E, X\right)<\varepsilon$, where $g / E(F)=g(E \cap F)$ for $E$ and $F \in S$. We shall denote by a $g / E$-refinement sum of a partition $\pi$, a sum of the form $\Sigma_{i \leqq n} f\left(x_{i}\right) g / E\left(E_{i}\right)$ where $\left[E_{i} ; i \leqq n\right]$ is a partition of $X$ in $S$ such that for each $i \leqq n 1$ ) $x_{i} \in E_{i}$ and (2) there exists (uniquely) an element $F$ in $\pi$ such that $E_{i} \subset F$. Since $\int_{X} f d g$ exists, $\int_{X} f d(g / E)=\int_{E} f d g$ exists and, hence, there exists a partition $\pi$ of $X$ in $S$ such that any two $g / E$ refinement sums of $\pi$ differ by less than $\varepsilon$. This implies that any two $g^{+}$-refinement sums of $\pi$ differ by less than $2(2\|f\| \cdot \varepsilon)+\varepsilon$. Hence $\int_{X} f d g^{+}$exists and, consequently, $\int_{X} f d V(g, E)$ exists.

Theorem 3.1. If $f$ is a partitionable (hence bounded) B-valued function on $X$ and $g \in H(X, S)$ then $f \in m(X, S, g, B)$, i.e., $f$ is also $g$-measurable, if an only if $f$ is Stieltjes integrable with respect to $g$.

Proof. If $f \in m(X, S, g, B)$ then $\int_{X} f d g$ exists. Hence it is sufficient to show that if $f$ is Stieltjes integrable with respect to $g$, then $f$ is $g$ measurable; moreover, in view of Lemma 3.1, it is sufficient to suppose $g \geqq 0$. Suppose $f \notin m(X, S, g, B)$. Then there exists $\varepsilon>0$ such that if $\pi=\left[E_{i} ; i \leqq n\right]$ is a partition of $X$ in $S$ then $g\left(\bigcup\left[E_{i} ; 0\left(f, E_{i}\right) \geqq \varepsilon\right]\right) \geqq \varepsilon$. 
Since $f$ is partitionable, there exists a partition $\left[F_{j} ; j \leqq m\right]$ of $X$ such that $\operatorname{lub}_{j \leqq m} 0\left(f, F_{j}\right)<\varepsilon / 8$. Let $\pi$ be a partition of $X$ in $S$. Let $\left[G_{k} ; k \leqq p\right]$ be an enumeration of the elements $E_{i}$ of $\pi$ such that $0\left(f, E_{i}\right) \geqq \varepsilon$. Let $H_{i j}=\bigcup\left[G_{k} ; G_{k} \cap F_{i} \neq \theta, G_{k} \cap F_{j} \neq \theta\right.$, and $d\left(F_{i}, F_{j}\right)=\inf [\| f(x)-$ $\left.\left.f(y) \| ; x \in F_{i}, y \in F_{j}\right] \geqq 3 \varepsilon / 4\right]$. Then $\bigcup H_{i j}=\bigcup G_{k}$ and, hence, there exists $H_{i j}$ such that $g\left(H_{i j}\right) \geqq \varepsilon /\left[m(m-1)\left(2^{-1}\right)\right]$. Let $x_{i} \in F_{i}$; let $x_{j} \in F_{j}$; and if $G_{k} \subset H_{i j}$ then let $x_{k i} \in G_{k} \cap F_{i}$ and $x_{k j} \in G_{k} \cap F_{j}$. Then

$$
\begin{aligned}
& \left\|\Sigma_{k} f\left(x_{k i}\right) g\left(G_{k}\right)-\Sigma_{k} f\left(x_{k j}\right) g\left(G_{k}\right)\right\| \\
& =\left\|\Sigma_{k}\left[\left(f\left(x_{k i}\right)-f\left(x_{i}\right)\right)+\left(f\left(x_{i}\right)-f\left(x_{j}\right)\right)+\left(f\left(x_{j}\right)-f\left(x_{k j}\right)\right)\right] g\left(G_{k}\right)\right\| \\
& \quad \geqq\left\|f\left(x_{i}\right)-f\left(x_{j}\right)\right\| \Sigma_{k} g\left(G_{k}\right)-\Sigma_{k}\left[\left\|f\left(x_{k i}\right)-f\left(x_{i}\right)\right\|+\| f\left(x_{j}\right)\right. \\
& \left.\quad-f\left(x_{k j}\right) \|\right] g\left(G_{k}\right) \geqq 3 \varepsilon / 4 \Sigma_{k} g\left(G_{k}\right)-\Sigma_{k}[\varepsilon / 8+\varepsilon / 8] g\left(G_{k}\right)=\varepsilon / 2 \Sigma_{k} G_{k} \\
& =\varepsilon / 2 g\left(H_{i j}\right) \geqq(\varepsilon / 2)\left(\varepsilon /\left[m(m-1)\left(2^{-1}\right)\right]=\varepsilon^{2} / m(m-1) .\right.
\end{aligned}
$$

Thus there exist refinement sums of $\pi$ which differ by at least $\varepsilon^{2} / m(m-1)$ and, hence ( $\pi$ was an arbitrary partition of $X$ in $S$ ), $f$ is not Stieltjes integrable with respect to $g$.

Let us now see to what extent Theorem 3.1 goes through for bounded vector valued functions which are not partitionable. If $f$ is $g$-measurable then the Stieltjes integral $\int_{x} f d g$ exists; however, the converse is not, in general, true. In fact, the following example shows that $f$ may be Stieltjes integrable with respect to a nontrivial bounded finitely additive set function $g$ even though $f$ oscillates "wildly" on every nonempty element of $S$. Let $X$ be the set of positive integers; let $S$ be the algebra of "periodic" subsets of $X: E \in S$ if and only if there exists a positive integer $n$ and a finite set $\left[i_{j} ; j \leqq k\right]$ of positive integers such that 1$)$ $i_{i} \leqq n$ for $j \leqq k$ and 2) $E=\bigcup_{j \leqq k}\left[i_{j}+m \cdot n ; m \geqq 0\right]$; and let $g\left(\bigcup_{j \leqq k}\left[i_{j}+\right.\right.$ $m \cdot n ; m \geqq 0])=k / n$. (Each element of $S$ has invariant Banach measure (cf. [7]); indeed, any Banach measure is an extension of $g$.) Let $B$ be the Banach space of bounded real valued functions on $X$ (sup norm). Let $\left[E_{i} ; i \geqq 1\right]$ be an enumeration of the nonempty elements of $S$ (S is a countable algebra of subsets of $X$ ). Let $R=\left[r_{i} ; i \geqq 1\right]$ be an enumeration of the rational numbers in the half open interval $(0,1]$. Since each nonempty element $E_{i}$ of $S$ is an infinite subset of $X$ and each of $X, R$, and $S$ is countable, there exists a real valued function $y$ on $X$ such that, for each nonempty element $E_{i}$ of $S, y\left(E_{i}\right)=[y(x)$; $\left.x \in E_{i}\right]=R$ and, hence, $0\left(y, E_{i}\right)=1$ for $i \geqq 1$. For each $x \in X$, let $f_{x}$ be the element of $B$ such that $f_{x}(x)=y(x)$ and $f_{x}(z)=0$ if $z \neq x$. For each $x \in X$, let $f(x)=f_{x}$. Hence $f$ is a bounded $B$-valued function on $X,\|f\|=1$, and $\int_{X} f d g$ exists and is the zero function. But, the oscillation of $f$ on each nonempty element $E_{i}$ of $S$ is one.

The function $y$ defined in the preceding paragraph is a bounded real valued function on $X$ which has the property that if $g \in H(X, S)$ 
and $g \neq 0$, then $\int_{X} f d g$ does not exist.

In his 1927 paper ([4]) on Riemann type integration of vector valued functions defined on an interval $[a, b]$, L. M. Graves gives an interesting example of an everywhere discontinuous vector valued function which is (Riemann type) integrable. Graves' example can be translated into the setting of our $\S 4$. In this setting Graves' example yields another example of a bounded non-measurable vector valued function which is Stieltjes integrable.

We shall now turn our attention to proving our principal theorems.

Definition 3.1. Let $T=[E ; E \subset X ; \chi(E) \in m(X, S, g)]$ where we denote by $\chi(E)$ the characteristic function of $E$.

Theorem 3.2 says, in effect, that $T$ is an algebra of subsets of $X$, $m(X, S, g, B)=C(X, T, B)$ (for all $B)$, and, hence, $m^{*}(X, S, g)=C^{*}(X, T)=$ $H(X, T)$. Hence we wish to characterize the elements of $T$ in a way which will enable us to show (1) $T$ is an algebra of subsets of $X$ and (2) one can approximate the elements $f$ of $m(X, S, g, B)$ uniformly by $(X, T, B)$-simple functions. In order to motivate our final characterization of $T$, we shall first give an elementary characterization of $T$ (Lemma 3.2) and then modify this orginal characterization in stages.

Lemma 3.2. If $E \subset X$, then $E \in T$ if and only if for each $\varepsilon>0$ there exist elements $H$ and $K$ of $S$ such that $H \subset E, K \subset E^{\prime}$, and $V\left(g,(H \cup K)^{\prime}\right)<\varepsilon$. (We denote complement $X-E$ of the subsets $E$ of $X$ by $E^{\prime}$.)

Proof. Necessity. If $E \in T$ and $\varepsilon>0$ then there exists a partition $\pi$ of $X$ in $S$ such that if $\left[F_{i} ; i \leqq n\right]$ is a refinement of $\pi$ in $S$ and each of $x_{i}$ and $y_{i}$ is an element of $F_{i}$ then $\left|\sum_{i \leqq n}\left(\chi(E)\left(x_{i}\right)-\chi(E)\left(y_{i}\right)\right) g\left(F_{i}\right)\right|<$ $\varepsilon / 2$ and, hence, $V\left(g, \cup\left[F_{i} ; F_{i} \cap E \neq \theta\right.\right.$ and $\left.\left.F_{i} \cap E^{\prime} \neq \theta\right]\right) \leqq \varepsilon / 2<\varepsilon$. Let $H=\cup\left[F_{i} ; F_{i} \subset E\right]$ and let $K=\cup\left[F_{i} ; F_{i} \subset E^{\prime}\right]$. Sufficiency. Let $\varepsilon>0$. Then there exist elements $H$ and $K$ of $S$ such that $H \subset E, K \subset E^{\prime}$, and $V\left(g,(H \cup K)^{\prime}\right)<\varepsilon$. Let $\pi=\left[H, K,(H \cup K)^{\prime}\right]$. If $\left[F_{i} ; i \leqq n\right]$ is a refinement of $\pi$ in $S$ and $x_{i} \in F_{i}$, then $\left|\sum_{i \leqq n} \chi(E)\left(x_{i}\right) g\left(F_{i}\right)-g(H)\right| \leqq$ $V\left(g,(H \cup K)^{\prime}\right)<\varepsilon$.

Lemma 3.3. If $E \subset X$, then $E \in T$ if and only if there exists a sequence $\left\{\left(E_{i}, F_{i}\right)\right\}$ of pairs of elements of $S$ such that $\left\{E_{i}\right\} \uparrow$ in $S,\left\{F_{i}\right\} \uparrow$ in $S,\left(\cup E_{i}\right) \cap\left(\cup F_{\imath}\right)=\theta, \lim _{i} V\left(g,\left(E_{i} \cup F_{i}\right)^{\prime}\right)=0, \cup E_{i} \subset E$ and $\cup F_{i} \subset E^{\prime}$.

Proof. Necessity. For each positive integer $i$ there exist, by Lemma 3.2, elements $H_{i}$ and $K_{i}$ of $S$ such that $H_{i} \subset E, K_{i} \subset E^{\prime}$, and $V\left(g,\left(H_{i} \cup K_{i}\right)^{\prime}\right)<2^{-i}$. Let $E_{i}=\bigcup_{j \leqq i} H_{j}$ and let $F_{i}=\bigcup_{j \leqq i} K_{j}$. 
Sufficiency follows from Lemma 3.2.

At this point we shall introduce some notation.

DeFINITION 3.2. Let

$$
\begin{aligned}
A & =\left[\left\{\left(E_{i}, F_{i}\right)\right\} ;\left\{E_{i}\right\} \uparrow \text { in } S,\left\{F_{i}\right\} \uparrow \text { in } S,\left(\cup E_{i}\right) \cap\left(\cup F_{i}\right)\right. \\
& \left.=\theta, \lim V\left(g, E_{i}^{\prime} \cap F_{i}^{\prime}\right)=0\right], \\
Q & =\left[\cup E_{i} ;\left\{\left(E_{i}, F_{i}\right)\right\} \in A\right],
\end{aligned}
$$

and

$$
D=\left[E ; E \subset\left(\cap\left(E_{i}^{\prime} \cap F_{i}^{\prime}\right)\right),\left\{\left(E_{i}, F_{i}\right)\right\} \in A\right] .
$$

The following lemma lists a few elementary properties of the sets $A, Q$, and $D$ which we shall have occasion to use.

LemMa 3.4.

(i ) $\left\{\left(E_{i}, F_{i}\right)\right\} \in A$ if and only if $\left\{\left(F_{i}, E_{i}\right)\right\} A$,

(ii) $S \subset Q$,

(iii) If each of $E$ and $F \in Q$ then each of $E \cup F$ and $E \cap F \in Q$,

(iv) $H \in D$ if and only if there exists a sequence $\left\{H_{i}\right\} \downarrow$ in $S$ such that $H \subset\left(\cap H_{i}\right)$ and $\lim _{i} V\left(g, H_{i}\right)=0$, and

(v) $D$ is an ideal in the algebra of all subsets of $X$.

Proof. (i) and (ii) are immediate consequences of Definition 3.2. (iii) Suppose each of $\left\{\left(K_{i}, L_{i}\right)\right\}$ and $\left\{\left(M_{i}, N_{i}\right)\right\} \in A$. Then $\left[\cup\left(K_{i} \cup M_{i}\right)\right] \cap$ $\left[\cup\left(L_{i} \cap N_{i}\right)\right]=\theta$ and $\left(K_{i} \cup M_{i}\right)^{\prime} \cap\left(L_{i} \cap N_{i}\right)^{\prime}=\left[\left(K_{i}^{\prime} \cap M_{i}^{\prime}\right) \cap\left(L_{i}^{\prime} \cup N_{i}^{\prime}\right)\right] \subset$ $\left[\left(K_{i}^{\prime} \cap L_{i}^{\prime}\right) \cup\left(M_{i}^{\prime} \cap N_{i}^{\prime}\right)\right]$ and, hence, $\left\{\left(\left(K_{i} \cup M_{i}\right),\left(L_{i} \cap N_{i}\right)\right)\right\} \in A$. (iv) Suppose $\left\{H_{i}\right\} \downarrow$ in $S, \lim _{i} V\left(g, H_{i}\right)=0$ and $H \subset\left(\cap H_{i}\right)$. Then $\left\{\left(H_{i}^{\prime}, \theta\right)\right\} \in A$. (v) follows immediately from (iv) and the fact that if $\left\{H_{i}\right\} \downarrow$ in $S,\left\{K_{i}\right\} \downarrow$ in $S, \lim _{i} V\left(g, H_{i}\right)=0, \lim _{i} V\left(g, K_{i}\right)=0, H \subset\left(\cap H_{i}\right)$, and $K \subset\left(\cap K_{i}\right)$, then $\left\{H_{i} \cup K_{i}\right\} \downarrow$ in $S, \lim _{i} V\left(g, H_{i} \cup K_{i}\right)=0$, and $(H \cup K) \subset\left(\cap\left(H_{i} \cup K_{i}\right)\right)$.

Turning again to our characterizations of $T$, we have the following.

Lemma 3.5. If $E \subset X$, then $E \in T$ if and only if there exist $\left\{\left(E_{i}, F_{i}\right)\right\} \in A$ and $K \in D$ such that $E=\left(\cup E_{i}\right) \cup K$.

Proof. Necessity follows from Lemma 3.3.

Sufficiency. Since $K \in D$, there exists a sequence $\left\{G_{i}\right\} \downarrow$ in $S$ such that $K \subset \cap G_{i}$ and $\lim _{i} V\left(g, G_{i}\right)=0$. Then $\left\{\left(E_{i} \cap G_{i}^{\prime}, F_{i} \cap G_{i}^{\prime}\right)\right\} \in A$, since $\left(\left(E_{i} \cap G_{i}^{\prime}\right) \cup\left(F_{i} \cap G_{i}^{\prime}\right)\right)^{\prime}=\left(E_{i}^{\prime} \cup G_{i}\right) \cap\left(F_{i}^{\prime} \cup G_{i}\right)=\left(E_{i}^{\prime} \cap F_{i}^{\prime}\right) \cup G_{i}$, and, hence by Lemma 3.3, $E \in T$.

Lemma 3.6. $T$ is an algebra of subsets of $X$. 
Proof. It is sufficient to show that $[H \cup K ; H \in Q, K \in D]$ is an algebra of subsets of $X$. Suppose each of $H$ and $H_{1} \in Q$ and $K$ and $K_{1} \in D$. Then $H \cup H_{1} \in Q$ and $K \cup K_{1} \in D$ and, hence, $(H \cup K) \cup\left(H_{1} \cup K_{1}\right)=$ $\left(H \cup H_{1}\right) \cup\left(K \cup K_{1}\right)$ is in $T$. In order to complete a proof of (vi), it is sufficient to show that if $H \in Q$ and $K \in D$, then $(H \cup K)^{\prime} \in T$. Suppose $\left\{\left(E_{i}, F_{i}\right)\right\} \in A,\left\{G_{i}\right\} \downarrow$ in $S, \lim _{i} V\left(g, G_{i}\right)=0$, and $K \subset\left(\cap G_{i}\right)$. Let $L=K-$ $\cup E_{i}$. Then $E_{i}^{\prime} \cap\left(F_{i} \cap G_{i}^{\prime}\right)^{\prime}=\left[E_{i}^{\prime} \cap\left(F_{i}^{\prime} \cup G_{i}\right)\right] \subset\left[\left(E_{i}^{\prime} \cap F_{i}^{\prime}\right) \cup G_{i}\right]$ which implies $\left\{\left(E_{i}, F_{i} \cap G_{i}^{\prime}\right)\right\} \in A$ and, hence $\left(\left(\cup E_{i}\right) \cap K\right)^{\prime}=\left(\left(\cup E_{i}\right) \cup L\right)^{\prime}=$ $\left[\cup\left(F_{i} \cap G_{i}^{\prime}\right)\right] \cup\left[\left(\cup\left(F_{i} \cap G_{i}^{\prime}\right)\right)^{\prime} \cap\left(\cup E_{i}\right)^{\prime} \cap L^{\prime}\right] \in T$.

At this point let us pause for a moment and survey the situation. We know that $T$ is the largest algebra $W$ (with respect to inclusion) of subsets of $X$ such that $C(X, W, B) \subset m(X, S, g, B)$. Hence, in order to complete a proof of Theorem 3.2, it is sufficient to prove the following

Lemma 3.7. $m(X, S, g, B) \subset C(X, T, B)$.

Proof. It is sufficient to show that if a function $f \in m(X, S, g, B)$ and $\varepsilon>0$, then there exists a partition $\left[E_{j}\right.$; $\left.\leqq P\right]$ of $X$ in $T$ such that $\max \left[0\left(f, E_{j}\right) ; j \leqq P\right]<\varepsilon$. Suppose a function $f \in m(X, S, g, B)$ and $\varepsilon>0$. Then there exists a partition $\left[E(1, t) ; t \leqq n_{1}\right]$ of $X$ in $S$ such that $0(f, E(1, t))<\varepsilon / 3$ for $i<n_{1}$ and $V\left(g, E\left(1, n_{1}\right)\right)<2^{-1}$. There exists a partition $\left[E(2, i) ; i \leqq n_{2}\right]$ of $E\left(1, n_{1}\right)$ in $S$ such that $0(f, E(2, i))<\varepsilon / 3$ for $i<n_{2}$ and $V\left(g, E\left(2, n_{2}\right)\right)<2^{-2}$. If we repeat this process inductively, we obtain a sequence $\left\{\left[E(i, t) ; t \leqq n_{i}\right]\right\}_{i \geqq 1}$ such that

(1) $\left[E(1, t) ; t \leqq n_{1}\right]$ is a partition of $X$ in $S$,

(2) $\left[E(i+1, t) ; t \leqq n_{i+1}\right]$ is a partition of $E\left(i, n_{i}\right)$ in $S$,

(3) $0(f, E(i, t))<\varepsilon / 3$ if $i \geqq 1$ and $t<n_{i}$, and

(4) $0\left(f, E\left(i, n_{i}\right)\right)<2^{-i}$.

Since $f$ is partitionable, there exists a finite partition $\left[F_{j} ; j \leqq P\right]$ of $X$ such that $\max \left[0\left(f, F_{j}\right) ; j \leqq P\right]<\varepsilon / 3$. If $i \geqq 1$ and $t<n_{i}$, let $F(E(i, t))=\min \left[j ; j \leqq P, E(i, t) \cap F_{j} \neq \theta\right]$. Let $V_{j}=\bigcup_{i} \bigcup_{t<n_{i}}[E(i, t)$; $F(E(i, t))=j]$, let $W=\cap E\left(i, n_{i}\right)$, and let $W_{j}=W \cap F_{j}$. For each positive integer $j \leqq P, V_{j} \in Q$ (if $V_{j k}=\bigcup_{i \leqq k} \bigcup_{t<n_{i}}[E(i, t) ; F(E(i, t))=j]$ and $F_{j k}=X-\left(V_{j k} \cup E\left(k, n_{k}\right)\right)$, then $\left\{\left(V_{j k}, F_{j k}\right)\right\}_{k \geqq 1} \in A$ and $\left.V_{j}=\bigcup_{k} V_{j k}\right)$ and $W_{j} \in D$. Hence, if we let $E_{j}=V_{j} \cup W_{j}$, then $\left[E_{j} ; j \leqq P\right]$ is a partition of $X$ in $T$ (Lemma 3.4) and $\max \left[0\left(f, E_{j}\right) ; j \leqq P\right]<\varepsilon$.

We note that $T$ is determined by $g$ and, hence, is independent of $B$; in fact, $T$ is the $g$-outer measure completion of $S$.

Therefore we now have the following

Theorem 3.2. Let $(X, S)$ be a set algebra, $g \in H(X, S)$, and $B$ be a Banach space. Then $m(X, S, g, B)=C(X, T, B)$.

In the case of real valued functions (i.e. $B=\mathscr{R}$ ) we know (cf. [5]) 
that $C^{*}(X, T)$ is isomorphic and isometric to $H(X, T)$ and hence

CoROLlaRY 3.2.1. If $g \in H(X, S)$ then $m^{*}(X, S, g)$ is isomorphic and isometric to $H(X, T)$ in the sense that if $L \in m^{*}(X, S, g)$ then there exists, uniquely, $h \in H(X, T)$ such that $L(f)=\int_{X}$ fdh for each $f \in m(X, S, g)$ and $\|L\|=\|h\|=V(h, X)$.

Moreover, this identification enables us to apply P. Porcelli's results (cf. [6]) on weak convergence and sequential weak compactness in $H(X, T)$ to obtain results such as the following

CoRollary 3.2.2. If $\left\{L_{n}\right\}$ is a sequence of elements of $m^{*}(X, S, g)$, then $\left\{L_{n}\right\}$ is weakly convergent if and only if $\lim _{n}\left(\sum_{i \geqq 1} L_{n} \chi\left(E_{i}\right)\right)$ exists for every sequence $\left\{E_{i}\right\}$ of pairwise disjoint elements of $T$.

We shall conclude this section by presenting an analog of Corollary 3.2.1 for an arbitrary Banach space $B$. However, in order to motivate our representation of $m^{*}(X, S, g, B)$ we shall first reinterpret Corollary 3.2.1 as follows. Corollary 3.2.1 says, in effect, that if $L \in C^{*}(X, T)$ then there exists, uniquely, $h \in H(X, T)$ such that $L(f)=\int_{X} f d h=$ $\lim _{\pi}\left(\sum_{x_{i} \in E_{i} \in \pi} f\left(x_{i}\right) h\left(E_{i}\right)\right)$ for each $f \in m(X, S, g)$ and $\|L\|=\|h\|$, where the limit is taken (cf. [5]) in the Moore-Smith sense over the directed set of partitions $\pi$ of $X$ in $T$. But, and this is the crux of the matter, if $F \in \mathscr{R}^{*}$ then $F(r)=F(1) \cdot r$ for each $r \in \mathscr{R}$. We exploit this fact as follows. Let $G$ be a function on $T$ to $\mathscr{R}^{*}$ such that if $E \in T$ and $r \in \mathscr{R}$ then $G(E)(r)=r \cdot h(E)$. Then

$$
\lim _{\pi}\left(\sum_{x_{i} \in E_{i} \in \pi} G\left(E_{i}\right)\left(f\left(x_{i}\right)\right)\right)=\lim _{\pi}\left(\sum_{x_{i} \in E_{i} \in \pi} f\left(x_{i}\right) h\left(E_{i}\right)\right) .
$$

Definition 3.3. Let $(X, S)$ be a set algebra and $B$ be a Banach space. Then we denote by $H(X, S, B)$ the Banach space of functions $G$ on $S$ to $B^{*}$ such that

(1) $G(\theta)=0$,

(2) If $E, F \in S$ and $E \cap F=\theta$, then $G(E \cup F)=G(E)+G(F)$, and

(3) $\|G\|=\sup _{\pi}\left(\sum_{E_{i} \in \pi}\left\|G\left(E_{i}\right)\right\|\right)<\infty$.

Moreover, if $f$ is a $B$-valued function on $X, G \in H(X, S, B)$, and $\lim _{\pi}\left(\sum_{x_{i} \in E_{i} \in \pi} G\left(E_{i}\right) f\left(x_{i}\right)\right)$ exists then we denote this limit by $\int_{X} d G \cdot f$.

The following two lemmas are immediate consequences of Definition 3.3.

LEMMA 3.8. If $\int_{X} d G \cdot f$ exists, then 


$$
\left|\int_{X} d G \cdot f\right| \leqq\|G\| \cdot \sup _{x \in X}|f(x)|
$$

LEMmA 3.9. If $f \in C(X, S, B)$ and $G \in H(X, S, B)$ then $\int_{X} d G \cdot f$ exists.

Lemma 3.10. If $G \in H(X, S, B)$ and $L(f)=\int_{X} d G \cdot f$ for each $f \in$ $C(X, S, B)$ then $L \in C^{*}(X, S, B)$ and $\|L\|=\|G\|$.

Proof. By Lemmas 3.8 and 3.9, $L \in C^{*}(X, S, B)$ and $\|L\| \leqq\|G\|$. Let $\varepsilon>0$. Then there exists a partition $\pi=\left[E_{i} ; i \leqq n\right]$ of $X$ in $S$ such that $\|G\|-\sum_{E_{i} \in \pi}\left\|G\left(E_{i}\right)\right\|<\varepsilon / 2$. For each $E_{i} \in \pi$ there exists $b_{i} \in B$ such that $\left\|b_{i}\right\|=1$ and $\left\|G\left(E_{i}\right)\right\|-G\left(E_{i}\right)\left(b_{i}\right)<\varepsilon / 2 n$. Let $f \sum_{i \leqq n} b_{i} \chi\left(E_{i}\right)$. Then $\|f\|=1$ and $L(f)=\int_{X} d G \cdot f=\sum_{i \leqq n} G\left(E_{i}\right)\left(b_{i}\right)>\|G\|-\varepsilon$ and, hence, $\|L\| \geqq\|G\|$.

Now we are ready to establish the following.

TheOREM 3.3. If $(X, S)$ is a set algebra and $B$ is a Banach space, then $C^{*}(X, S, B)$ is isomorphic and isometric to $H(X, S, B)$. Moreover, if $L \in C^{*}(X, S, B)$ then there exists, uniquely, $G \in H(X, S, B)$ such that $L(f)=\int_{X} d G \cdot f$ for each $f \in C(X, S, B)$ and $\|G\|=\|L\|$.

Proof. In view of Lemma 3.10 it is sufficient to prove the "moreover" part of Theorem 3.3. Let $L \in C^{*}(X, S, B)$. Let $G$ be the function on $S$ to $B^{*}$ such that $G(E)(b)=L(b \chi(E))$ for each $E \in S$ and $b \in B$. Then $G(\theta)=0$. Moreover, if $E, F \in S, E \cap F=\theta$, and $b \in B$, then $G(E \cup F)$ $(b)=L(b \chi(E \cup F))=L(b \chi(E))+L(b \chi(F))=G(E)(b)+G(F)(b)$. Finally, if $\left[E_{i} ; i \leqq n\right]$ is a partition of $X$ in $S, b_{i} \in B$ for $i \leqq n$, and $\left\|b_{i}\right\| \leqq 1$ for $i \leqq n$, then $\sum_{i \leqq n} G\left(E_{i}\right)\left(b_{i}\right)=\sum_{i \leqq n} L\left(b_{i} \chi\left(E_{i}\right)\right)=L\left(\sum_{i \leqq n} b_{i} \chi\left(E_{i}\right)\right) \leqq\|L\|$. Thus (we can conclude from the argument used in Lemma 3.10) $\sum_{i \leqq n}\left\|G\left(E_{i}\right)\right\| \leqq\|L\|$ and, hence, $G \in H(X, S, B)$. Moreover, if $k$ is an $(X, S, B)$-simple function, then $L(k)=\int_{X} d G \cdot k$. Hence, since the $(X$, $S, B)$-simple functions are dense in $C(X, S, B), L(f)=\int_{X} d G \cdot f$ for each $f \in C(X, S, B)$ (if $k$ is an $(X, S, B)$-simple function then

$$
\begin{aligned}
& \left|L(f)-\int_{X} d G \cdot f\right| \\
& \quad=\left|L(f-k)+\int_{X} d G \cdot(k-f)\right| \leqq(\|L\|+\|G\|)\|f-k\| \\
& \quad=2\|L\| \cdot\|f-k\|) .
\end{aligned}
$$

As an immediate consequence of Theorems 3.2 and 3.3 , we have 
the following.

THEOREM 3.4. If $(X, S)$ is a set algebra, $g \in H(X, S)$, and $B$ is a Banach space, then $m^{*}(X, S, g, B)$ is isomorphic and isometric to $H(X, T, B)$ in the sense of Theorem 3.3 (i.e. if $L \in m^{*}(X, S, g, B)$ then there exists, uniquely, $G \in H(X, T, B)$ such that $L(f)=\int_{X} d G \cdot f$ for each $f \in m(X, S$, $g, B)$ and $\|G\|=\|L\|)$.

4. A Banach space of Riemann integrable functions. In this section we give a determination of the bounded linear functionals on the Banach space $R[a, b]$.

We denote $[a, b]$ by $I$ and $R[a, b]$ by $R(I)$. Let $S_{I}$ denote the algebra of subsets of $I$ generated by segments $(c, d)$ where $a \leqq c<d \leqq b$ (i.e. $E \in S_{I}$ if and only if there exists a finite subset $F$ of $I$ and a finite set $\left[\left(c_{i}, d_{i}\right) ; a \leqq c_{i}<d_{i} \leqq b\right]$ of segments such that $E=F \cup\left(\bigcup_{i \leqq n}\left(c_{i}, d_{i}\right)\right)$. Then we denote by $s$ the element of $H\left(I, S_{I}\right)$ such that if $a \leqq c<d \leqq$ $b$ then $s((c, d))=d-c$ and if $x \in I$ then $s([x])=0$ (i.e. if $E \in S_{I}$ then $s(E)$ is the Jordan content of $E)$. Finally, if $f$ is a real valued function on $I$ and $x \in I$, let $d(f, x)=\lim _{\varepsilon \rightarrow 0^{+}} 0(f,(x-\varepsilon, x+\varepsilon) \cap I)$.

Let us recall that a real valued function $f$ on $I$ is in $R(I)$ if and only if (1) $f$ is bounded and (2) if $\varepsilon>0$ then there exists a finite set $\left[\left(c_{i}, d_{i}\right) ; i \leqq n\right]$ of segments such that $[x ; d(f, x) \geqq \varepsilon] \subset \bigcup_{i \leqq n}\left(c_{i}, d_{i}\right)$ and $\sum_{i \leqq n} s\left(\left(c_{i}, d_{i}\right) \cap I\right)<\varepsilon$. Hence $R(I)=m\left(I, S_{I}, s\right)=C\left(I, T_{I}\right)$ where $T_{I}$ is determined as in Theorem 3.2. Moreover, $R^{*}(I)$ is isomorphic and isometric to $H\left(I, T_{I}\right)$ in the sense of Theorem 3.4.

T. H. Hildebrandt informed the author that 0 . Frink had shown (cf. [3], in particular Theorem 10) that a real valued function $f$ on $I$ is Riemann integrable if and only if there exists a sequence $\left[f_{i} ; f_{i}=\right.$ $\sum_{j \leqq n_{i}} \boldsymbol{r}_{i j} \chi\left(E_{i j}\right), r_{i j} \in \mathscr{R}, E_{i j}$ is measurable Jordan: the boundary of $E_{i j}$ has Jordan content zero] of simple functions such that $\lim _{i}\left(\sup _{x \in I} \mid f(x)-\right.$ $\left.f_{i}(x) \mid\right)=0$. From our characterizations of $T_{I}$, we see that $E \in T_{I}$ if and only if there exist sequences $\left\{E_{i}\right\} \uparrow$ in $S$ and $\left\{F_{i}\right\} \uparrow$ in $S$ such that $\cup E_{i} \subset E$, $\cup F_{i} \subset E^{\prime}$ and $\lim _{i} s\left(\left(E_{i} \cup F_{i}\right)^{\prime}\right)=0$; moreover, since points have $s$-measure zero we can require that the sets $E_{i}$ and $F_{i}$ be open. Thus $E \in T_{I}$ if and only if $E$ is measurable Jordan (the interior of $E \supset \cup E_{i}$ and the interior of $E^{\prime} \supset \cup F_{i}$; the boundary of $E$ is a closed set). Hence (cf. [5]) every bounded linear functional $L$ on $R(I)$ is expressible in the form $\int_{X} f d g$ where the integral is of the Stieltjes type, $g$ is bounded finitely additive set function on the algebra $T_{I}$ of subsets of $I$ which have Jordan content, and the total variation of $g$ on $I$ is $\|L\|$.

We shall conclude by discussing (briefly) Riemann type integration of partitionable vector valued functions defined on $I$. In the case of partitionable functions $f$ defined on $I$ both the norm definition of Riemann 
integrability of $f$ defined by Graves (cf. [4]) and the refinement definiton of Riemann integrability of $f$ are equivalent to the $S$-integrability of $f$ with respect to $s$. We shall not prove this statement; however, we shall prove Lemma 4.1 which provides a tool with which to reduce a proof of this statement to a standard argument.

LEMMA 4.1. Let $f$ be a $B$ valued function on $X, g$ be a nonnegative finitely additive set function on $S, b \in B$, and $\pi$ be a partition of $X$ in S. Then

$$
\begin{aligned}
& \sup _{\pi^{\prime} \geqq \pi \text { in } S}\left(\left\|b-\sum_{y_{j} \in F_{j} \in \pi^{\prime}} f\left(y_{j}\right) g\left(F_{j}\right)\right\|\right) \\
& \quad \leqq \sup _{\pi}\left(\left\|b-\sum_{x_{i} \in E_{i} \in \pi} f\left(x_{i}\right) g\left(E_{i}\right)\right\|\right) .
\end{aligned}
$$

Proof. It is sufficient to show that if $w \in B, F$ and $G \in S, F \cap G=$ $\theta, E=F \cup G, y \in F$, and $z \in G$, then there exists $x \in E$ such that $\| w-$ $f(x) g(E)\|\geqq\| w-(f(y) g(F)+f(z) g(G)) \|$ (i.e. we can eliminate one set at a time). It is sufficient to suppose that $g(E) \neq 0$ and $\|w-f(z) g(E)\| \leqq$ $\|w-f(y) g(E)\|$. Then

$$
\begin{aligned}
& \|w-(g(F) f(y)+g(G) f(z))\| \\
& \quad=\|g(F) / g(E)(w-g(E) f(y))+g(G) / g(E)(w-g(E) f(z))\| \\
& \quad \leqq g(F) / g(E)\|w-g(E) f(y)\|+g(G) / g(E)\|w-g(E) f(z)\| \\
& \quad \leqq\|w-g(E) f(y)\| .
\end{aligned}
$$

Thus we are in a position to assert that the most general linear functional on the space of partitionable $B$-valued Riemann integrable functions defined on $I$ is determined by a bounded finitely additive $B^{*}$ valued set function defined on the algebra $T_{I}$ of subsets of $I$ which have Jordan content.

\section{BIBLIOGRAPHY}

1. R. B. Darst, A note on abstract integration, Trans. Amer. Math. Soc., 99 (1961), 292297.

2. Dunford and Schwartz, Linear Operators, Interscience, New York, 1958.

3. O. Frink, Jordan measure and Riemann integration, Annals of Math., Ser. 2, 34 (1933), 518-526.

4. L. M. Graves, Riemann integration and Taylor's theorem in general analysis, Trans. Amer. Math. Soc., 29 (1927), 163-177.

5. T. H. Hildebrandt, On bounded linear functional operations, Trans. Amer. Math. Soc., 36 (1934), 868-875.

6. P. Porcelli, Two embedding theorems with applications to weak convergence and compactness in spaces of additive type functions Indiana Journal, 9 (1960), 273-292.

7. P. Porcelli and P. M. Anselone, On invariant Banach measures, Indiana Journal, 10 (1961), 309-316. 



\title{
PACIFIC JOURNAL OF MATHEMATICS
}

\author{
EDITORS
}

Ralph S. Phillips

Stanford University

Stanford, California

M. G. Arsove

University of Washington

Seattle 5, Washington
A. L. Whiteman

University of Southern California Los Angeles 7, California

Lowell J. Paige

University of California

Los Angeles 24, California

\section{ASSOCIATE EDITORS}
E. F. BECKENBACH
D. DERRY
M. OHTSUKA
H. L. ROYDEN
E. SPANIER
E. G. STRAUS
T. M. CHERRY
F. WOLF

\section{SUPPORTING INSTITUTIONS}

\author{
UNIVERSITY OF BRITISH COLUMBIA \\ CALIFORNIA INSTITUTE OF TECHNOLOGY \\ UNIVERSITY OF CALIFORNIA \\ MONTANA STATE UNIVERSITY \\ UNIVERSITY OF NEVADA \\ NEW MEXICO STATE UNIVERSITY \\ OREGON STATE UNIVERSITY \\ UNIVERSITY OF OREGON \\ OSAKA UNIVERSITY \\ UNIVERSITY OF SOUTHERN CALIFORNIA
}

\author{
STANFORD UNIVERSITY \\ UNIVERSITY OF TOKYO \\ UNIVERSITY OF UTAH \\ WASHINGTON STATE UNIVERSITY \\ UNIVERSITY OF WASHINGTON \\ $*$
AMERICAN MATHEMATICAL SOCIETY \\ CALIFORNIA RESEARCH CORPORATION \\ SPACE TECHNOLOGY LABORATORIES \\ NAVAL ORDNANCE TEST STATION
}

Mathematical papers intended for publication in the Pacific Journal of Mathematics should be typewritten (double spaced), and the author should keep a complete copy. Manuscripts may be sent to any one of the four editors. All other communications to the editors should be addressed to the managing editor, L. J. Paige at the University of California, Los Angeles 24, California.

50 reprints per author of each article are furnished free of charge; additional copies may be obtained at cost in multiples of 50 .

The Pacific Journal of Mathematics is published quarterly, in March, June, September, and December. Effective with Volume 13 the price per volume (4 numbers) is $\$ 18.00$; single issues, $\$ 5.00$. Special price for current issues to individual faculty members of supporting institutions and to individual members of the American Mathematical Society: $\$ 8.00$ per volume; single issues $\$ 2.50$. Back numbers are available.

Subscriptions, orders for back numbers, and changes of address should be sent to Pacific Journal of Mathematics, 103 Highland Boulevard, Berkeley 8, California.

Printed at Kokusai Bunken Insatsusha (International Academic Printing Co., Ltd.), No. 6, 2-chome, Fujimi-cho, Chiyoda-ku, Tokyo, Japan.

PUBLISHED BY PACIFIC JOURNAL OF MATHEMATICS, A NON-PROFIT CORPORATION

The Supporting Institutions listed above contribute to the cost of publication of this Journal, but they are not owners or publishers and have no responsibility for its content or policies. 


\section{Pacific Journal of Mathematics}

\section{Vol. 12, No. $2 \quad$ February, 1962}

William George Bade and Robert S. Freeman, Closed extensions of the Laplace operator determined by a general class of boundary conditions . . . . . . . 395

William Browder and Edwin Spanier, H-spaces and duality ............. 411

Stewart S. Cairns, On permutations induced by linear value functions . . . . . . . 415

Frank Sydney Cater, On Hilbert space operators and operator roots of

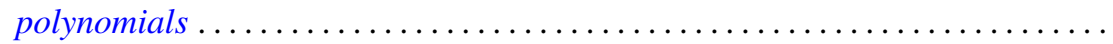

Stephen Urban Chase, Torsion-free modules over $K[x, y] \ldots \ldots \ldots \ldots \ldots \ldots \ldots 437$

Heron S. Collins, Remarks on affine semigroups . . . . . . . . . . . . . . . . 449

Peter Crawley, Direct decompositions with finite dimensional factors . . . . . . . 457

Richard Brian Darst, A continuity property for vector valued measurable

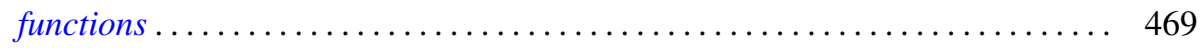

R. P. Dilworth, Abstract commutative ideal theory ................. 481

P. H. Doyle, III and John Gilbert Hocking, Continuously invertible spaces . . . . . . 499

Shaul Foguel, Markov processes with stationary measure . . . . . . . . . . . 505

Andrew Mattei Gleason, The abstract theorem of Cauchy-Weil ............ 511

Allan Brasted Gray, Jr., Normal subgroups of monomial groups . . . . . . . . . . 527

Melvin Henriksen and John Rolfe Isbell, Lattice-ordered rings and function

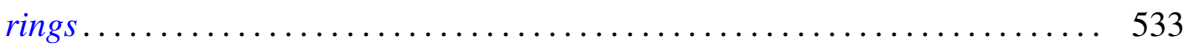

Amnon Jakimovski, Tauberian constants for the $[J, f(x)]$ transformations . ..... 567

Hubert Collings Kennedy, Group membership in semigroups . . . . . . . . . . . 577

Eleanor Killam, The spectrum and the radical in locally $m$-convex algebras ..... 581

Arthur H. Kruse, Completion of mathematical systems . . . . . . . . . . . . . 589

Magnus Lindberg, On two Tauberian remainder theorems ................ 607

Lionello A. Lombardi, A general solution of Tonelli's problem of the calculus of

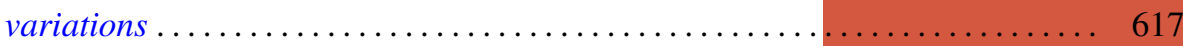

Marvin David Marcus and Morris Newman, The sum of the elements of the powers

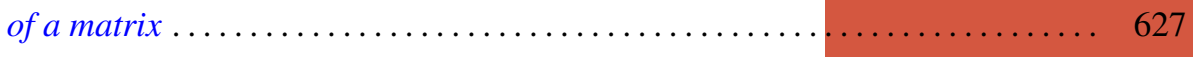

Michael Bahir Maschler, Derivatives of the harmonic measures in

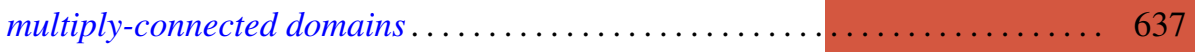

Deane Montgomery and Hans Samelson, On the action of $\mathrm{SO}(3)$ on $S^{n} \ldots \ldots \ldots 649$

J. Barros-Neto, Analytic composition kernels on Lie groups . . . . . . . . . . . . 661

Mario Petrich, Semicharacters of the Cartesian product of two semigroups ...... 679

John Sydney Pym, Idempotent measures on semigroups . . . . . . . . . . . . 685

K. Rogers and Ernst Gabor Straus, A special class of matrices . . . . . . . . . . . . 699

U. Shukla, On the projective cover of a module and related results . . . . . . . . . 709

Don Harrell Tucker, An existence theorem for a Goursat problem . . . . . . . . . . . 719

George Gustave Weill, Reproducing kernels and orthogonal kernels for analytic

differentials on Riemann surfaces ......................... 729

George Gustave Weill, Capacity differentials on open Riemann surfaces ........ 769

G. K. White, Iterations of generalized Euler functions . . . . . . . . . . . . . 777

Adil Mohamed Yaqub, On certain finite rings and ring-logics . . . . . . . . . 785 